\title{
Desarrollo de un sistema integrado de sensores para la caracterización organoléptica del pisco
}

\author{
Joan Calzado ${ }^{1}$, German Comina ${ }^{1}$ y José Solís ${ }^{1,2}$ \\ ${ }^{1}$ Facultad de Ciencias, Universidad Nacional de Ingeniería, Av. Tupac Amaru 210, Lima 25, Perú \\ ${ }^{2}$ Instituto Peruano de Energia Nuclear, Av. Canada 1470, Lima 41, Perú
}

Recibido 8 enero 2011 - Aceptado 30 marzo 2011

\begin{abstract}
Hemos construido un sistema integrado de sensores para la caracterización organoléptica de un licor destilado de la vid llamado pisco, analizando su fase líquida en una celda electroquímica, ademas se analiza la fase gaseosa en una pequeña cámara donde se encuentran los sensores de gas. Se ha diseñado una nariz electrónica con 4 sensores de gas de diferentes características (Figaro Inc.) y una lengua electrónica basada en una celda electroquímica de dos electrodos de acero inoxidable con $\mathrm{Pt}$ y Au, respectivamente. El sistema está compuesto principalmente por sensores de gas, actuadores, válvulas, bombas de líquido y gas que están gobernadas por el microcontrolador PIC18F4523 que se encarga del envío de datos hacia el computador. El prototipo desarrollando es robusto, económico y portátil. Para el tratamiento de datos se ha desarrollado un programa en Matlab usando análisis por componentes principales. Se ha realizado pruebas con diferentes piscos, lográndose obtener la huella digital de los piscos analizados.
\end{abstract}

Palabras claves: Matlab, LabView, microcontrolador, voltametría, sensor de gas..

\section{Development of an integrated system of sensors for organoleptic characterization of pisco}

We have built an integrated system of sensors for organoleptic characterization of a liquor distilled from grapes called pisco analyzing its liquid phase in an electrochemical cell, in addition is analyzed its gas phase in a small chamber with the gas sensors. We have designed an electronic nose with 4 gas sensors with different characteristics (Figaro Inc.) and an electronic tongue based on an electrochemical cell with two electrodes of stainless steel with $\mathrm{Pt}$ and $\mathrm{Au}$, respectively. The system is composed mainly of gas sensors, actuators, valves, liquid and gas pumps that are governed by the PIC18F4523 microcontroller which also are responsible for sending data to the computer. The prototype developed is robust, economical and portable. For data processing we have developed a program in Matlab using principal components analysis. We have tested with different piscos obtaining interesting fingerprints of the pisco.

Keywords: Matlab, LabView, microcontroller, voltmetry, gas sensor..

\section{Introducción}

Durante los últimos años la calidad del pisco ha adquirido una gran importancia porque es el valor añadido del producto. La investigación del pisco y sus constituyentes es un tema que ha adquirido una importancia, especialmente para los enólogos. Generalmente el estudio de estos compuestos se lleva a cabo utilizando técnicas complejas como la cromatografía de gases y la espectrometría de masas. Se han identificado más de 54 compuestos volátiles en los piscos chilenos[1] usando cromatografía de gases multidimensional, no se ha en- contrado trabajo similar para el caso del pisco peruano. Sin embargo la caracterización organoléptica del pisco, como son color, olor y sabor, dependen esencialmente de los componentes presentes en su fabricación y es realizada por personal profesional llamados como catadores.

La Norma Técnica Peruana 211.001.2006 da los requisitos organolépticos del pisco. Esta caracterización se realiza por personas especialmente entrenadas que utilizan principalmente sus sentidos del olfato y gusto. La nariz y lengua humana tienen una gran cantidad de células olfativas y del gusto que están en el epite- 
lio olfativo y diseminado por la lengua, respectivamente. Lo que uno toma o huele estimula a estas células que envían información a través del sistema nervioso al cerebro, donde se compara con patrones almacenados y se reconoce el sabor o aroma. En los últimos años se han desarrollado sistemas electrónicos que tratan de imitar los sentidos del ser humano, estos sistemas son mucho más elementales e imperfectos. El concepto de nariz electrónica como sistema inteligente no apareció realmente hasta 1982[2] y el de lengua electrónica alrededor de 1990[3]. Una definición generalmente aceptada de un sistema denominado nariz electrónica puede ser: instrumento que comprende de una agrupación de sensores químicos con sensibilidades parcialmente solapadas junto a un sistema de reconocimiento de patrones, capaz de analizar y reconocer aromas simples o complejos.

Los sensores químicos no son específicos para una analito, al contrario, su selectividad muestra un solapamiento parcial para diferentes especies. Esto hace que se puede extraer información importante de la muestra. Entonces la combinación de sensores no-específicos juntamente con un análisis de datos multivariados nos da una herramienta poderosa para determinar atributos antes que parámetros específicos. Los sistemas olfativos[4-7] y de gusto[8-10] artificiales se han utilizado para la caracterización del vino con muy buenos resultados. Como un degustador utiliza principalmente los sentidos del olfato y gusto, se ha fusionado ambos sistemas para mejorar la discriminación del sistema principalmente para alimentos[11]. La combinación de estos sistemas también se han usado satisfactoriamente para la caracterización del vino[12-14]. Para el caso del pisco, se han logrado discriminar tres tipos de pisco con una nariz electrónica[15].

En este artículo, reportamos el desarrollo de un sistema integrado de sensores para la caracterización organoléptica del pisco, básicamente es la combinación de una nariz y una lengua electrónica que podría lograr una mejor discriminación de los piscos. La función del prototipo desarrollado no es analizar cuantitativamente los aromas complejos sino obtener información cualitativa del conjunto. Es decir obtener, analizar y reconocer huellas de diferentes piscos.

\section{Prototipo desarrollado}

En la Figura 1 se muestra el diagrama del prototipo que se ha desarrollado. Este consta de una nariz y una lengua electrónica que se ha desarrollado usando un microcontrolador PIC 16F877, sensores de gas y electrodos. El sistema manipula el líquido y el gas a analizar mediante pequeñas bombas. Se lleva el aroma a una cámara donde están los sensores de gas y el líquido a una cámara donde está la celda electroquímica con electrodos de Au y Pt. El sistema se comunica con una computadora usando el puerto RS232. Además se ha desarrollado un programa para el tratamiento de datos en Matlab. El corazón del prototipo es el microcontrolador, ya que se requiere controlar varios procesos. A continuación se describirá las partes del prototipo.

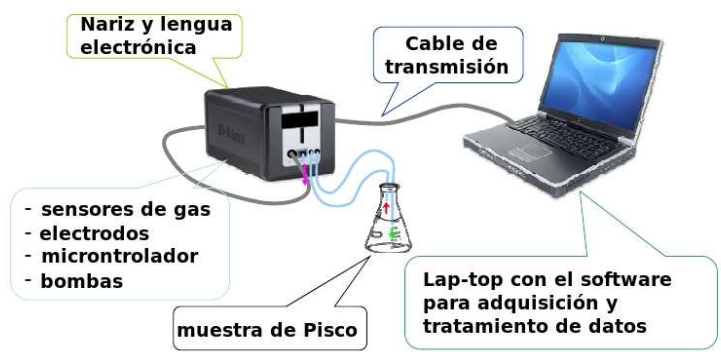

Figura 1: Diagrama del prototipo desarrollando.

\subsection{Nariz Electrónica}

Los sensores de gas de estado sólido están basados en óxidos semiconductores cuya resistencia cambia cuando la concentración de gases en el medio ambiente cambia[17]. Generalmente el material sensible que se utiliza en los sensores de gas de la compañía Figaro (TGS) es el $\mathrm{SnO}_{2}$. Cuando el $\mathrm{SnO}_{2}$ se mantiene a alta temperatura $\left(400{ }^{\circ} \mathrm{C}\right)$ en el aire, iones de oxigeno se adsorben (O-, O-2) en la superficie del material con una carga negativa. Los electrones del material que están en la superficie del cristal son atrapados por el oxigeno al ser adsorbido, produciéndose una capa superficial cargada positivamente. Entonces, se desarrolla una barrera Schottky, eVs, en los contactos intergranulares y controla la conductancia de un material poroso. Esta barrera de potencial previene que los portadores se muevan libremente. La presencia de gases reductores disminuye la densidad superficial de oxigeno, y la altura de la barrera, eVs, en el borde de grano se reduce. La reducción de la barrera disminuye la resistencia del sensor.

Para el prototipo se han utilizando 4 sensores de gas marca Figaro Inc., a continuación damos las especificaciones de los mismos:

TGS 813 Sensor de Gases Combustibles.

TGS 822 Sensor de Solventes Orgánicos.

TGS 825 Sensor de H2S.

TGS 826 Sensor de amonia.

Estos 4 sensores tienen diferentes sensibilidades, así que al montarlo en una cámara de acero (ver Fig. 2) donde el aroma ingresa cada sensor proporciona diferente respuesta. 


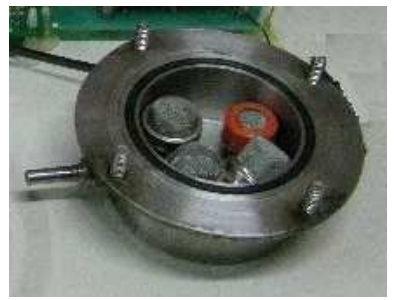

Figura 2: Cámara de la nariz electrónica diseñada y construida.

En la Figura 3 se muestra el diagrama de la nariz electrónica que se ha desarrollado. Se está utilizando una pequeña bomba de vacío marca Hargraves para hacer ingresar el aroma en la cámara donde están los sensores de gas.

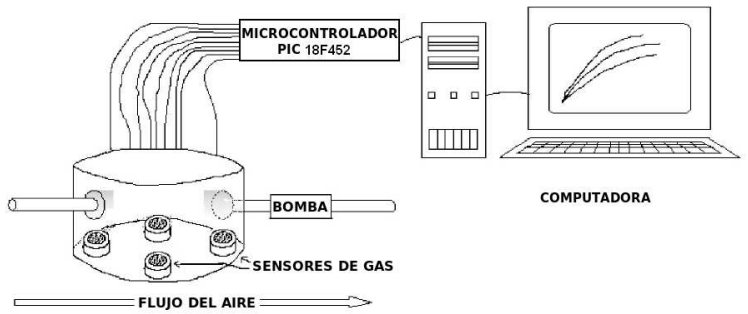

Figura 3: Diagrama de la nariz electrónica del prototipo desarrollado.

La variación de la resistencia eléctrica entre los pines de cada sensor provoca el cambio de voltaje en un circuito divisor. Estas variaciones de voltaje son medidos en cuatro puertos de conversión A/D del microcontrolador PIC18F4523 que posee 12 bits de resolución, lo cual fue, una de las principales razones por la que se eligió esta serie de entre otros microcontroladores de la familia Microchip.

\subsection{Lengua Electrónica}

Una lengua electrónica se basa en un arreglo de sensores no específicos para líquidos y un programa de reconocimiento de patrones. La lengua electrónica consta de una celda voltimétrica modificada con un arreglo de electrodos de trabajo de diferentes metales (oro y platino), un contraelectrodo que es el electrodo de referencia. Este arreglo está en contacto con el líquido, al aplicar una diferencia de potencial se mide la corriente que pasa a través de cada uno de los electrodos. La corriente que se mide es función de la naturaleza del líquido que se analiza y varía de acuerdo al contenido de los diferentes componentes electro-activos y especies cargadas que se encuentran en la solución[16]. Se pueden usar diferentes electrodos, dependiendo de la magnitud del potencial aplicado, el modo empleado como voltimetría pulsada o cíclica, y la actividad catalítica del electrodo. La voltimetría es una técnica electroquímica. Una celda para medidas voltimétricas de tres electrodos consiste del electrodo de trabajo (WE), un electrodo de referencia (RE) y un electrodo auxiliar (AE). La corriente debido a una reacción electroquímica se mide entre los electrodos auxiliar y de trabajo, el voltaje se aplica entre los electrodos de referencia y de trabajo. El rango del potencial aplicado puede variar y depende del material del electrodo y de la composición de la solución.

En la práctica, dos reacciones ocurren en el electrodo de trabajo. Uno involucra la corriente farádica o redox, que puede ser debido a la transferencia electrónica debido a la oxidación o reducción de las especies electroactivas a través de la interfase electrodo/solución. Este proceso obedece a la ley de Faraday cuando la corriente es proporcional a la cantidad de reacción química producida. La otra reacción en el electrodo involucra un proceso no farádico. Una carga capacitiva es obtenida cuando las especies polares o cargadas son distribuidas en una doble capa en la superficie del electrodo. Dependiendo de la magnitud del potencial aplicado, especies cargadas de signo opuesto son atraídos al electrodo, mientras que las otras son repelidas. Entonces, las corrientes de carga depende de la magnitud del potencial aplicado, área efectiva de contacto del electrodo, composición de la solución y la absorción/desorción.

Para el prototipo desarrollado se utilizó el sistema de 2 electrodos, en este caso se hizo un puente entre el electrodo de referencia y el electrodo auxiliar. Las especificaciones técnicas de los electrodos utilizados son:

- Electrodo auxiliar: Acero Inoxidable

- Electrodos de trabajo: uno de platino y el otro de oro

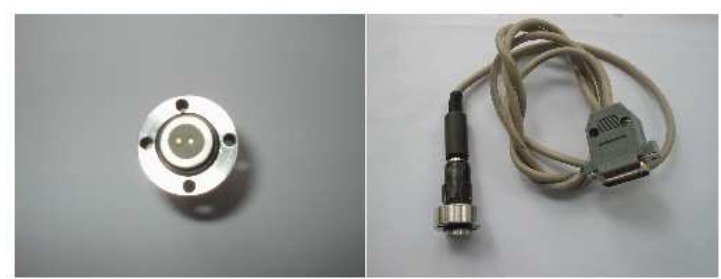

Figura 4: Electrodos diseñados para el prototipo de la lengua electrónica.

Los pares de electrodo de trabajo y auxiliar se han diseñado y desarrollado en el laboratorio. Se fabricó un cabezal sensor y la cámara para líquidos, ver Figura 4, dicho cabezal funciona como soporte para los electrodos de una celda electroquímica de dos electrodos. La base de soporte es de acero inoxidable y funciona como contra-electrodo, los electrodos de trabajo se fabricaron con alambre de oro (Premionß, 1mm de diámetro, 
con una pureza de $99.985 \%$ ) y platino (World Precision Instruments Inc, 1mm de diámetro, Item PTP406). El aislante que soporta a los electrodos de trabajo en la estructura del contraelectrodo es acrílico dental (3M ESPE Valux Plus). Se esta utilizando una pequeña bomba marca Hargraves para succionar el líquido a analizar e ingresarlo en la cámara donde están los electrodos.

Luego se ha diseñado y construido un potenciostato que proporciona los voltajes requeridos, y mide la corriente entre el electrodo de trabajo y contraelectrodo, esto se ha realizado con ayuda del DAC MCP4922 y un conversor ADC de 10 bits del microcontrolador. El diagrama de la lengua electrónica que se ha desarrollado se muestra en la Figura 5.

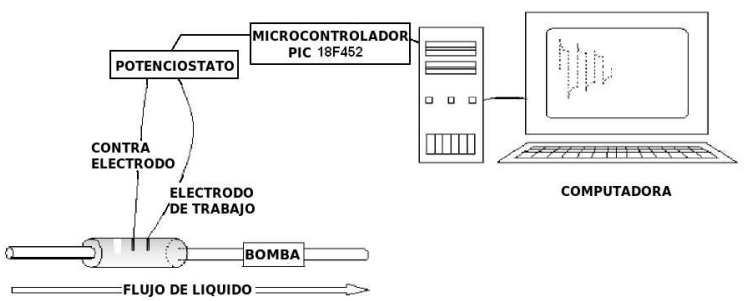

Figura 5: Diagrama de la componente lengua electrónica del prototipo que se ha desarrollado.

\subsection{Programa de reconocimiento de patro- nes}

Tres o más variables pueden tener relaciones de diversos tipos. Descriptivas como las que establecen perfiles, las de separación de grupos, las de segmentación, las de determinación de influencias eficientes entre varias variables. En general, se las clasifica en dos grandes grupos[19]:

1. Métodos explicativos: como regresión lineal, análisis discriminante, regresión logística, modelos de respuesta probit, logic, modelos loglineales, etc.

2. Métodos descriptivos: como análisis de conglomerados, análisis factorial, análisis de componentes principales, análisis de correspondencias simples y múltiples, etc.

Este tipo de análisis ha tenido un alto desarrollo y hoy ocupa el corazón del análisis estadístico avanzado. Desafortunadamente es exigente y poco amigable en comprensión matemática aunque lo es gráficamente. La gran virtud de este tipo de análisis es que sintetiza las relaciones entre las variables estudiadas, que de otro modo, tendrían que establecerse con los análisis anteriores, largos y que conllevan a equivocaciones.

El análisis de los datos que se integra a una nariz electrónica tiene su origen en la química analítica[20].
Debido a que es necesario comprimir y extraer toda información relevante del voluminoso conjunto de datos obtenidos, ya que en muchas ocasiones la información esencial no depende de variables aisladas sino de la interrelación entre las mismas. La técnica de análisis de componentes principales (Principal Component Analysis, PCA) es un método estadístico clásico. Este algoritmo cae dentro de las técnicas denominadas reconocimiento de patrones o técnicas de inteligencia artificial y permite reducir, representar y extraer información relevante al mismo tiempo[21]. Es una forma de identificar patrones en datos y expresarlos de tal manera que se resalte sus similitudes y diferencias. El PCA es un procedimiento matemático que transforma un gran número de variables que posiblemente estén correlacionadas en un número pequeño de variables no correlacionadas llamadas componentes principales. El algoritmo PCA suele ser clasificado como un método no supervisado de reconocimiento de patrones, ya que su uso más extendido con las narices electrónicas se limita a representar bidimensionalmente un conjunto de medidas para ver si se pueden formar agrupaciones (clusters) espontáneas entre las diferentes medidas realizadas. El primer componente principal toma en cuenta la máxima varianza en los datos, y las siguientes componentes toman en cuenta la máxima varianza posible remanente. La varianza es un estimador de la divergencia de una variable respecto del valor medio de todos los datos.

La medición de $j$ parámetros o variables de $i$ muestras u objetos, pueden representarse por la matriz: $X_{i j}$. Por tanto cada medición de una muestra, se expresa como un vector en el espacio $j$-dimensional. El PCA permite reducir las dimensiones de dicho espacio. Usando el PCA los datos del espacio $j$-dimensional, por lo general, se proyectan en el plano formado por la componente principal 1 ( $\mathrm{PC} 1$, es la dirección en el espacio $j$-dimensional, donde está la mayor variancia de $X$ ) y la componente principal 2 ( $\mathrm{PC} 2$, es la segunda dirección en importancia donde está la variancia de $X$ ); pero también es posible proyectar los puntos sobre el espacio formado por las primeras tres componentes principales.

El PCA usa el algoritmo Non-lineal Iteractive Projections by Alternating Least-Squares, NIPALS, inventado por Herman Wold en 1966. El primer paso es centrar cada objeto de la matriz respecto de la media $\bar{x}_{j}$ como se observa en la ecuación:

$$
x_{i j}=x_{i j}-\bar{x}
$$

Luego se crea un modelo lineal, el cual consta por un término llamado de estructura, formado por el producto de dos matrices $T P^{T}$, y el término de error residual, $E$, o la parte de la matriz que no pudo ajustarse al término de estructura, como se observa en la ecuación:

$$
X=T P^{T}+E
$$


O en forma equivalente:

$$
X=\sum_{w=1}^{A} t_{w} p_{w}^{T}+E
$$

donde $A$, es el número de componentes principales del modelo, $t_{w}$ es el vector de score para la componente $w$ y $p_{w}$ es el vector de loading para la componente $w$. El algoritmo NIPALS calcula sucesivamente cada $t_{w}, p_{w} \mathrm{y}$ cada $E_{w}$ que es la matriz de error para cada componente principal $w$. Los gráficos que se utilizan en el presente trabajo son los gráficos de score con dos componentes principales PC1 y PC2. El software de reconocimiento de patrones basado en el análisis multivariado se ha desarrollado usando funciones especiales en Matlabß.

\subsection{Sistema Integrado de Sensores para la caracterización organoléptica del Pisco}

La integración de la nariz y lengua electrónica se ha logrado colocándolos en una caja. Tanto la electrónica como las cámaras de medición y las bombas de succión se encuentran en la caja y solamente salen dos tubos, uno para la succión del vapor del pisco y otro para la succión del pisco (ver Fig. 6). En la laptop se tiene el programa de adquisión y procesamiento de datos, asi como el programa de analisis de componentes principales de reconocimiento de patrones.

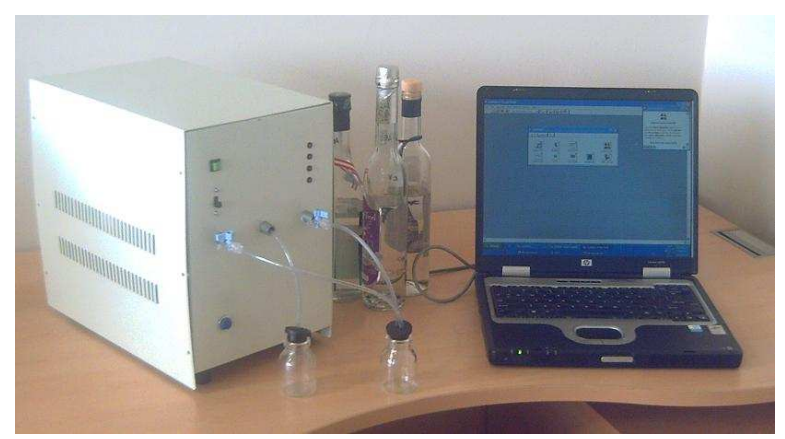

Figura 6: Fotografía del prototipo desarrollado conectado a una computadora portátil.

\section{Resultados}

Para las medidas con la nariz electrónica se usaron $3 \mathrm{~mL}$ de pisco y para la lengua electrónica se usaron 15 $\mathrm{mL}$. Para las pruebas con el prototipo desarrollado se escogieron los siguientes piscos:

DU0: Destilado de Uva (Fina Estirpe)

AR1: Pisco Aromático (Mosto Verde - Ocuaje)

AR2: Pisco Aromático (Viejo Tonel-MOSCATEL)
AR3: Pisco Aromático (PAYET)

PA1: Pisco Acholado (Quiñones)

PA2: Pisco Acholado (Bianca)

PA3: Pisco Acholado (Biondi)

PP1: Pisco Puro (Bianca)

PP2: Pisco Puro (Ocuaje)

PP3: Pisco Puro (Payet)

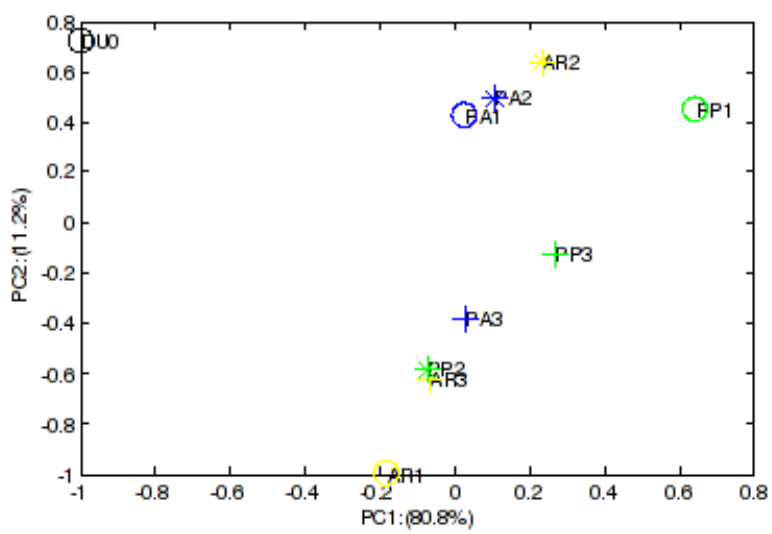

Figura 7: PCA normalizado de las muestras de pisco medidas por cromatografía de gases.

Para verificar que los piscos que se usaron tienen diferencias en cuanto a su composición se utilizó un cromatógrafo de gases marca Varian modelo 3800. Los parámetros del método son la temperatura del inyector, $250{ }^{\circ} \mathrm{C}$ ( $\left.5 \mathrm{~min}\right)$; la temperatura del horno, $60{ }^{\circ} \mathrm{C}$ $(0.25 \mathrm{~min}), 65^{\circ} \mathrm{C}$ a $5^{\circ} / \min (0 \min )$ y $200^{\circ} \mathrm{C}$ a $36^{\circ} / \mathrm{min}$ (0 min); la temperatura del detector, $280^{\circ} \mathrm{C}$ ( $\left.5 \mathrm{~min}\right)$; presión, 5 psi; flujo, $1.3 \mathrm{~mL} / \mathrm{min}$; split ratio, 50 . En la Figura 7 se distingue claramente los tipos de pisco, el destilado de uva es un pisco adulterado y se observa que está muy alejado de los otros piscos.

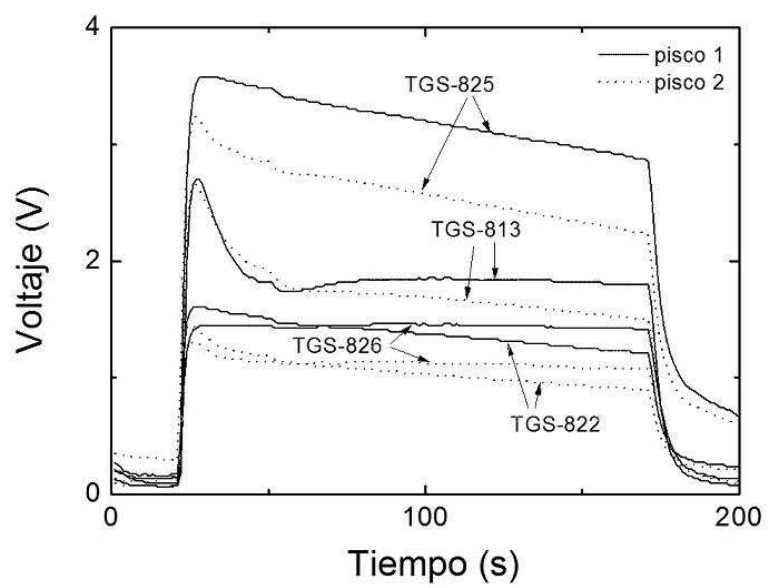

Figura 8: Respuesta de los cuatro sensores de gas a aromas de dos piscos. 
En la Figura 8 se observan la respuesta de los cuatro sensores de gas a dos tipos de pisco. Se puede ver que cada sensor tiene una respuesta característica. En la lengua electrónica cada electrodo también tiene respuesta característica para cada pisco (ver Fig. 10). En la Fig. 9 se muestra la voltimetría de -0.8 a $0.8 \mathrm{~V}$ de los electrodos de platino y oro a dos piscos diferentes. Se puede observar que las voltimetrías cíclicas son características de cada electrodo y del tipo de pisco.

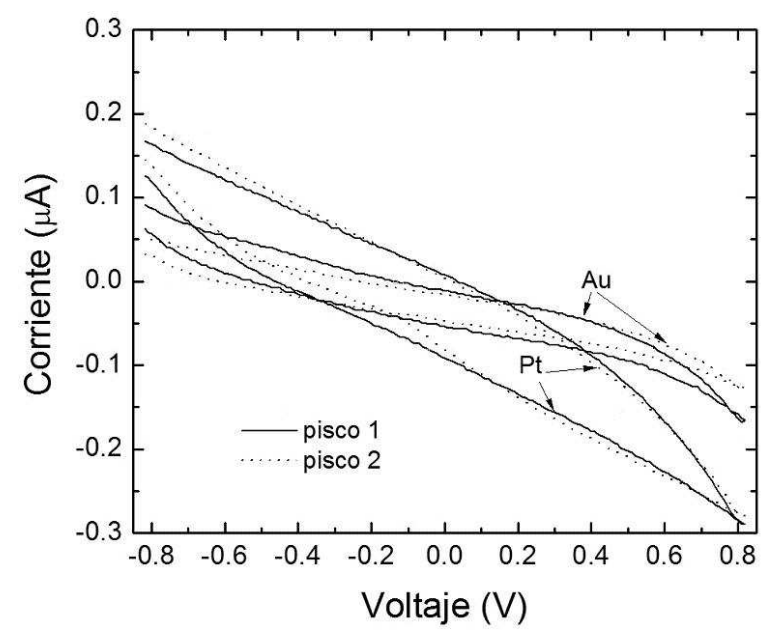

Figura 9: Voltimetría cíclica de los electrodos de $\mathrm{Au}$ y $\mathrm{Pt}$ a diferentes muestras de pisco.
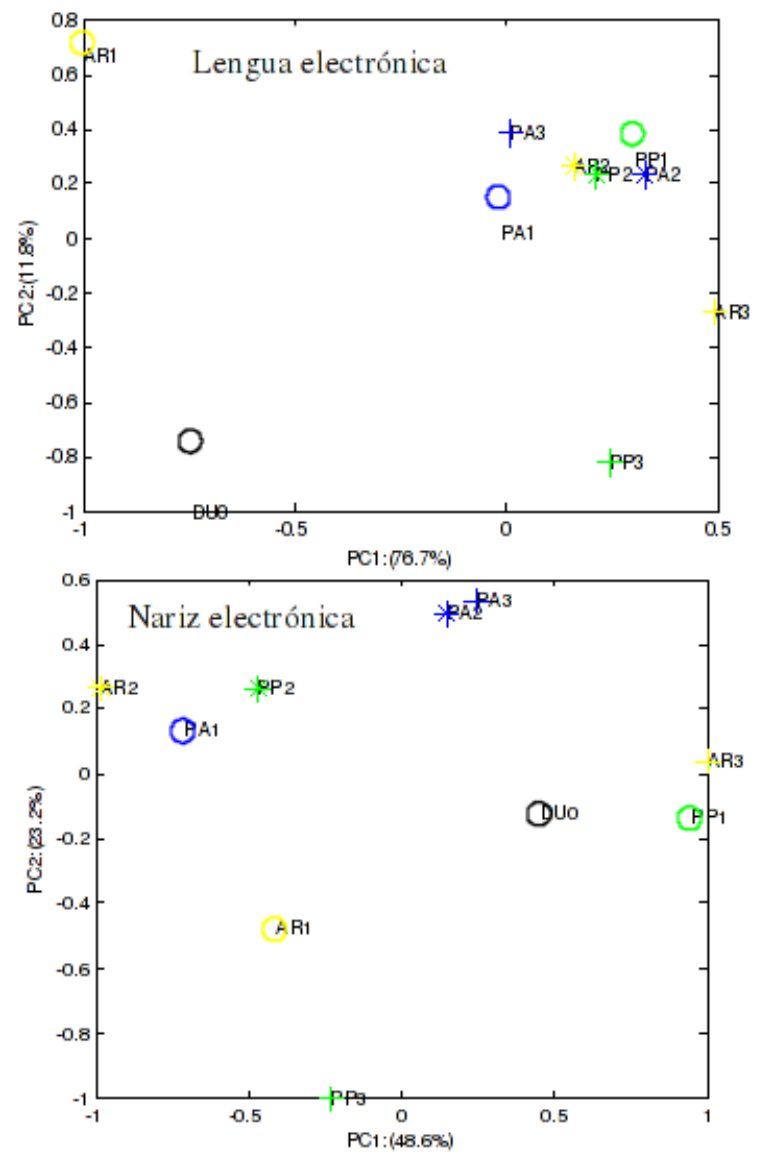

Figura 10: PCA normalizado de las muestras de pisco medidas con la nariz y lengua electrónica para diferentes piscos.

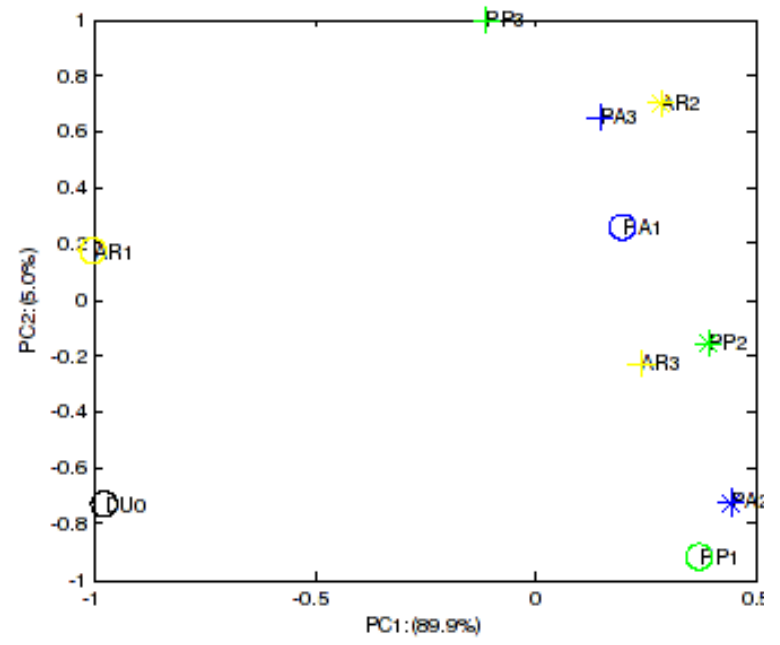

Figura 11: PCA normalizado de las muestras de pisco medidas con el sistema integrado de sensores.
En la Fig. 10 se muestra un PCA para cada componente del prototipo, se observa que la lengua discrimina muy bien los diferentes tipos de pisco, mientras que la nariz no lo hace muy bien. Sin embargo si integramos ambas medidas tenemos un gráfica donde se distingue claramente cada tipo de pisco (ver Fig. 11).

\section{Discusión}

El prototipo desarrollado ha logrado distinguir los diferentes piscos analizados, y podria realizar la caracterización organoléptica del pisco con una base de datos del o los piscos a analizar. Sin embargo para esto se tiene que extender las caracterizaciones a otros piscos y estudiar la reproducibilidad de los resultados. Este prototipo puede ser una herramienta para realizar el control de calidad de la producción del pisco, ya que no tiene limitación en cuanto al número de muestras a analizar. Para que el prototipo se pueda usar comer- 
cialmente se tiene que continuar optimizando, usar mas sensores de gas y otros electrodos. Mejorar la resolución de los datos utilizando PICs de 12 bits. Integrar los programas desarrollados y hacerlo mas amigable. Poner a prueba el prototipo en la cadena de producción del pisco, utilizarlo en diferentes etapas del mismo y ver si el equipo puede usarse en la producción del pisco. El prototipo desarrollado es de bajo costo y esta al alcance de pequeños productores.

\section{Conclusiones}

El prototipo desarrollado consta de componentes denominados nariz y lengua electrónica que son controlados por un microcontrolador. Se ha desarrollado los programas para realizar de una forma automática las medidas y el tratamiento de los datos usando análisis de componentes principales. Se han analizado diferentes piscos, lográndose discriminar los mismos.

\section{Referencias}

[1] M. Herraiz, G. Reglero, T. Herraiz y E. Loyola, Analysis of wine distillates made from muscat grapes (Pisco) by multidimensional gas chromatography and mass spectrometry, J. Agric. Food Chem. 38, 1540 (1990).

[2] J.W. Gardner y P.N. Barlett, Electronic noses: Principles and applications, Oxford University Press, Londres (1999).

[3] K. Hayashi, M. Yamanaka, K. Toko y K. Yamafuji, Multichannel taste sensor using lipid membranes, Sensors and Actuators B 2, 205 (1999).

[4] V. Ferreira, R. Lopez y J. F. Cacho, Quantitativedetermination of the odorants of young red wines from different grape varieties, J. Sci. Food Agric. 80, 1659 (2000).

[5] A. Guadarrama, J. A. Fernández, M. Iñiguez, J. Souto y J. A. de Saja, Discrimination of wine aroma using an array of conducting polymer sensors in conjunction with solid-phase micro-extraction (SP$M E)$ technique, Sensors and Actuators B 77, 401 (2001).

[6] M. Penza y G. Cassano, Chemometric characterization of Italian wines by thin-film multisensors array and artificial neural networks, Food Chem. 86, 283 (2004).

[7] J. Lozano, J.P. Santos, M. Aleixandre, I. Sayago, J. Gutierrez y M.C. Horrillo, Identification of typical wine aromas by means of an electronic nose, IEEE Sens. J. 6, 173 (2006).

[8] A. Legin, A. Rudnitskaya, Y. Vlasov, E. Di Natale, C. Mazzone y A. D'Amico. Application of electronic tongue for quantitative analysis of mineral water and wine. Electroanalysis 11, 814 (1999).

[9] V. Parra, T. hernando, M.L. Rodriguez-Mendez y J.A. de Saja, Electro-chemical sensor array made from bis-phthalocyanine modified carbon paste electrodes for discrimination of red wines, Electrochim. Acta 49, 5177 (2004).

[10] V. Parra, A.A. Arrieta, J. A. Fernandez-Escudero, H. Garcia, C. Apetrei, M. L. Rodriguez-Mendez y J. A. de Saja, E-tongue based on a hybrid array of voltammetric sensors based on phthalocyanines, perylene derivatives and conducting polymers: Discrimination capability towards red wines elaborated with different varieties of grapes, Sensors and Actuators B 115, 54 (2006). 11 F. Winquist, P. Wide y I. Lundstrom, The combination of an electronic tongue and an electronic nose for improved classification of fruit juices, Tech. Dig. of Eurosensors XII Conference, Southampton UK., Sept 13-16, 1998, IOP, Bristol (1998).

[11] S. Buratti, D. Ballabio, S. Benedetti y M.S. Cosio, Prediction of Italian red wine sensorial descriptors from electronic nose, electronic tongue and spectrophotometric measurements by means of Genetic Algorithm regression models, Food Chemistry 100, 211 (2007).

[12] C. Di Natale, R. Paolesse, A. Macagnano, A. Mantini, A. D'Amico, M. Ubigli, A. Legin, L. Lvova, A. Rudnitskaya y Y. Vlasov, Application of a combined artificial olfaction and taste system to the quantification of relevant compounds in red wine, Sensors and Actuators B 69, 342 (2000).

[13] C. Di Natale, R. Paolesse, M. Burgio, E. Martinelli, G. Pennazza y A. D'Amico, Application of metalloporphyrins-based gas and liquid sensor arrays to the analysis of red wine, Analytica Chimica Acta 513, 49 (2004).

[14] M.J. Madou y S.R. Morrison, Chemical Sensing With Solid State Devices, Academic Press, San Diego (1989).

[15] F. Winquist, P. Wide y I. Lundstrom, An electronic tongue based on voltammetry, Analytica Chimica Acta 357, 21 (1997). 
[16] M.J. Madou y R. Morrison, Chemical Sensing with Solid States Devices, Academic Press, Inc., San Diego (1989).

[17] C. Luyo, L. Reyes, J.L. Solis, J. Rodríguez, W. Estrada, I. Fábregas y R J. Candal, Morphology Tailored $\mathrm{SnO}_{2}$ Thin Films by Spray Gel Process, Thin Solid Films 516, 25 (2007).

[18] K. Esbensen, D. Guyot y F. Westad, Multivariate Data Analysis in Practice. An introduction to multivariate data analysis and experimental design, CAMO ASA, Trondheim (2000).

[19] K.S. Booksh, Encyclopedia of Analytical Chemistry, editado por R.A. Meyers, pp. 8145-8169, John Wiley \& Sons Ltd., Chichester (2000).

[20] J.E. Jackson, Principal component and factor analysis: Part 1 Principal components, J. Qual. Tech. 3, 1 (1981). 